\title{
Identification and mapping of induced chromosomal deletions using sequence polymorphisms
}

\author{
Emmanuel Vanrobays*, Barbara H. Jennings* ${ }^{*}$, and David Ish-Horowicz \\ Developmental Genetics Laboratory Cancer Research UK, LRI, London UK \\ BioTechniques 48:53-60 (January 2010) doi 10.2144/000113348 \\ Keywords: single-strand conformation polymorphism; transposon excision; reverse genetics; Drosophila; mapping \\ *E.V.'s present address is CNRS, UMR 6247 GReD, Université Blaise Pascal, INSERM U931, 24 avenue des Landais, 63177 Aubiére, France. \\ ${ }^{* *}$ B.H.J.'s present address is Transcriptional Regulation Laboratory, UCL Cancer Institute, London, WC1E 6BT, UK.
}

One of the many advantages of Drosophila melanogaster as a model organism is the relative ease with which gene deletions can be generated by imprecise excision of transposon insertions. Here, we describe a simple, fast, and efficient method of screening for single-gene excision events that is not biased by prior assumptions of the mutant phenotype. DNA sequence polymorphisms were used as co-dominant electrophoretic markers to identify candidate deletions in a single generation, and to delimit the breakpoints to within $0.5-1 \mathrm{~kb}$, thereby rapidly identifying deficiencies that affect only the gene of interest. In addition, we used polymorphism profiling to map existing deficiencies. The method can also be applied to map the extent of deletions generated by $\mathrm{x}$-rays and to identify targeted mutations generated by engineered zinc-finger nucleases in Drosophila and other polymorphic model organisms (e.g., zebrafish, mouse, Caenorhabditis elegans).

\section{Introduction}

Reverse genetics is a powerful approach for studying gene function in vivo. In Drosophila, a good model for studying eukaryotic gene function because of the wide range of available mutations and the relative lack of genetic redundancy, there are many different ways to selectively disrupt the function of genes of interest $(1,2)$. These include ends-out replacement gene targeting by homologous recombination (3), post-transcriptional knockdown via dsRNA (4), and zinc-finger nucleaseinduced targeting $(5,6)$, or by using sitedirected recombination between FRT sites in trans $(7,8)$. Perhaps the most straightforward method is imprecise excision of transposable elements inserted adjacent to the target gene (9). To date, transposon insertions have been identified for more than two-thirds of annotated genes in $D$. melanogaster, and this proportion is ever increasing due to several genome-wide transposon mutagenesis projects $(10,11)$.

Drosophila has several different types of transposable elements (including $P$, minos, and piggy Bac) $(7,11,12)$ whose transposases catalyze transposon excision by generating a double-strand break in the genomic DNA. Although this break is usually repaired precisely, the DNA break ends for $P$ and minos elements can be rejoined via non-homologous end joining (NHEJ), which causes deletions at the break site (13) that can be exploited to generate localized deficiencies.

The frequency of such imprecise events varies from locus to locus ( 1 in 5 to 1 in 100 excision events) (1,11), and the frequency of imprecise excision can be increased dramatically in some specific genetic backgrounds or by inhibiting pairing between the transposable element chromosome and its homolog $(14,15)$. Flies carrying a visibly marked [e.g., white ${ }^{+}\left(w^{+}\right)$] transposable element inserted into or near a gene of interest are crossed with flies containing a stable source of transposase. Flies in which the element has been excised are identified by loss of the visible marker (e.g., $w^{-}$) and can be tested individually for predicted or expected homozygous or hemizygous phenotypes.

The alternative to phenotypic assays is to identify imperfect excision events molecularly. This can be done by Southern blotting; however, this method is unsuitable for large-scale screening, and also misses deletions that remove the DNA probe region. Another technique is to use PCR on genomic DNA from heterozygous flies with flanking primer sites that are too distant to amplify a wild-type or transposon-containing fragment, but that would amplify a fragment made smaller by a suitable deletion. Such PCR screens are often inefficient because the amplified fragment-whose size cannot be predicted in advance-might be poorly resolved or visualized on the chosen gel system. The method can also fail if too large a footprint of the transposable element is still present on the chromosome, or if there are small but inactivating deletions.

Alternatively, one can use PCR on genomic DNA from homozygous flies, and screen for the loss of a genomic fragment. This method is efficient but relies on the homozygous mutants being viable, and requires extra generations to produce homozygous flies. Finally, quantitative, real-time PCR can be used to detect the reduced gene copy number (16), but it is technically challenging to screen a large number of flies for a two-fold difference in gene dosage.

Here, we report a simple, rapid, and efficient strategy to screen and map the extent of transposon-induced deletions of any locus of interest in a single generation using polymorphic sequence markers. This method, which makes no assumptions 
about the mutant phenotype and uses pretested primers, is based on the comparison of polymorphic sequences between the strain carrying the transposable element and an unrelated tester strain, and identifies all deletions in the polymorphic region. Imprecise excision events are revealed by selective loss of polymorphic alleles associated with the strain bearing the transposable element. All steps including the screening and the mapping can be achieved within a single generation, substantially reducing the time and stock maintenance required. We have validated this method by identifying single-gene deletions at two different loci in the Drosophila genome. This method can also be used to map deletions generated in other ways (including via $\mathrm{x}$-rays), or adapted for use with other polymorphic genetic model organisms.

\section{Materials and methods}

Drosophila stocks

All Drosophila stocks and crosses were set up on standard medium at $25^{\circ} \mathrm{C}$. The $w$; MKRS, $P\{\Delta 2-3\} / T M 6 B, T b, w ; T M 6 B, T b / S b$, w;P\{EPgy2\}HUS1-like ${ }^{E Y 14510}, w ; D f(3 R) E D_{50} 95 / T M 6 C, c u, S b$ (Deficiency for 82C5;82E7), Df(3L)Cat (75C1-75C2;75F1), and $y^{I}, w^{67223} ; P\{l a c W\} R p L 11^{k 16914} / C y O$ lines were obtained from the Bloomington Stock Center (Indiana University, Bloomington, IN, USA). $P\{G a w B\}^{N P 3550}$ was provided by the Drosophila Genetic Resource Center (DGRC; Kyoto Institute of Technology, Kyoto, Japan). We used isogenized parental chromosomes, although intrapopulation polymorphisms are relatively uncommon in inbred stocks predicted to be monomorphic (17).

\section{MICROSCOPE AUTOMATION}

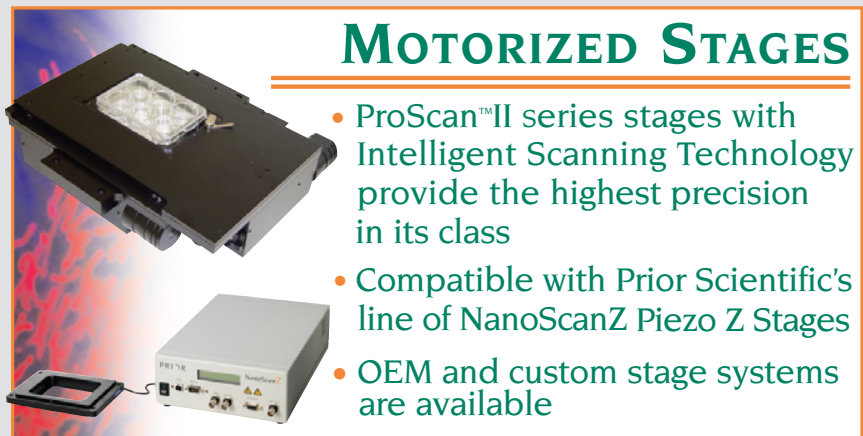

\section{FLUORESCENCE ILLUMINATION}

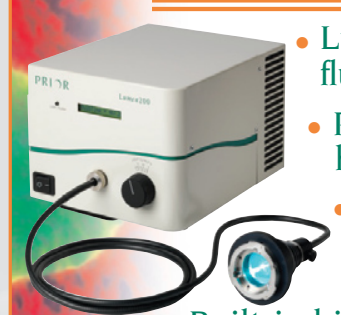

- Lumen 200/200Pro series 200 Watt fluorescence illumination systems

- Pre-centered bulb with 2,000 hour life

- Stabilized DC power supply eliminates variations in light intensity

Built-in high speed light attenuator and six position high speed filter wheel

\section{Where Vision Meets Precision}

A
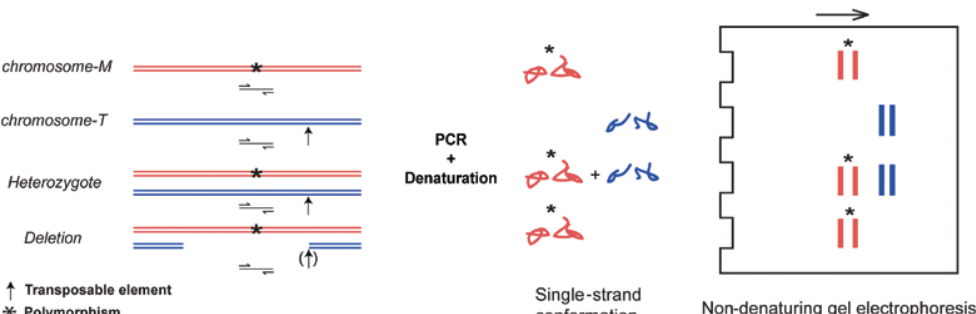

* Polymorphism

Non-denaturing gel electrophoresis

B

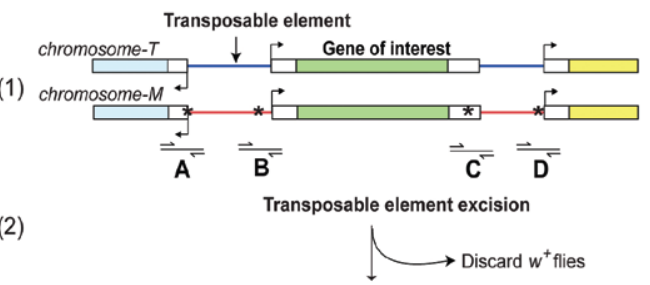

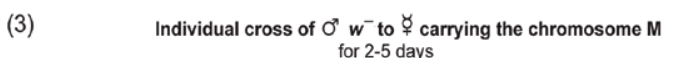

(4)

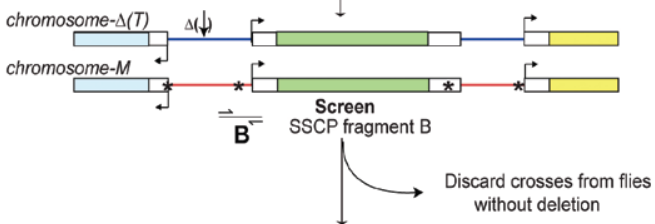

(5)

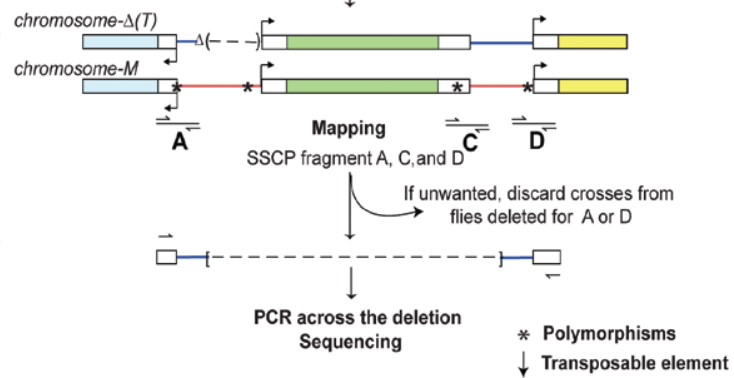

Figure 1. Principles of screening using polymorphisms as co-dominant markers. (A) SSCP analysis. After denaturation of the double-stranded DNA PCR product, the two single strands of DNA fold to form a secondary structure that depends on primary sequence. A polymorphism (indicated by an asterisk) leads to the formation of single-stranded structures that differ between the mapping chromosome (chromosome-M, in red) and the transposon-bearing chromosome (chromosome-T, in blue), resulting in differential mobility in non-denaturing gel electrophoresis. Both polymorphic forms remain after precise transposon excision, whereas imprecise excision on chromosome-T extending past and removing the polymorphic site shows only the chromosome-M profile. Semi-arrows represent primers delimiting amplified fragments. Vertical arrow indicates position of the $P$ element here and in Figure 1B. (B) Screen and mapping process: (1) Identification of polymorphisms between chromosome-T (blue) and chromosome-M (red). (2 and 3) Generation of excisions, and breeding the $w$ - flies heterozygous for newly generated excisions [chromosome- $\Delta(T)$ ] for recovery of the deletion. (4) DNA from individual mated heterozygotes is screened by SSCP for loss of a first polymorphism (fragment B) to identify flies with imprecise excisions extending in the desired direction. (5) Flies with such a deletion are mapped using other polymorphisms in and around the gene of interest (fragments $A, C$, and D) Single gene deletions should remove $B$ and $C$ but retain $A$ and $D$. (6) PCR amplification across the deletion for determining its precise extent by DNA sequencing. Intergenic regions are represented by a line. Genes are boxed. Semi-arrows correspond to the primers used to amplify polymorphic fragments. Polymorphisms are represented by asterisks. Excisions are symbolized by delta $(\Delta)$, and deleted regions by dashed lines. 

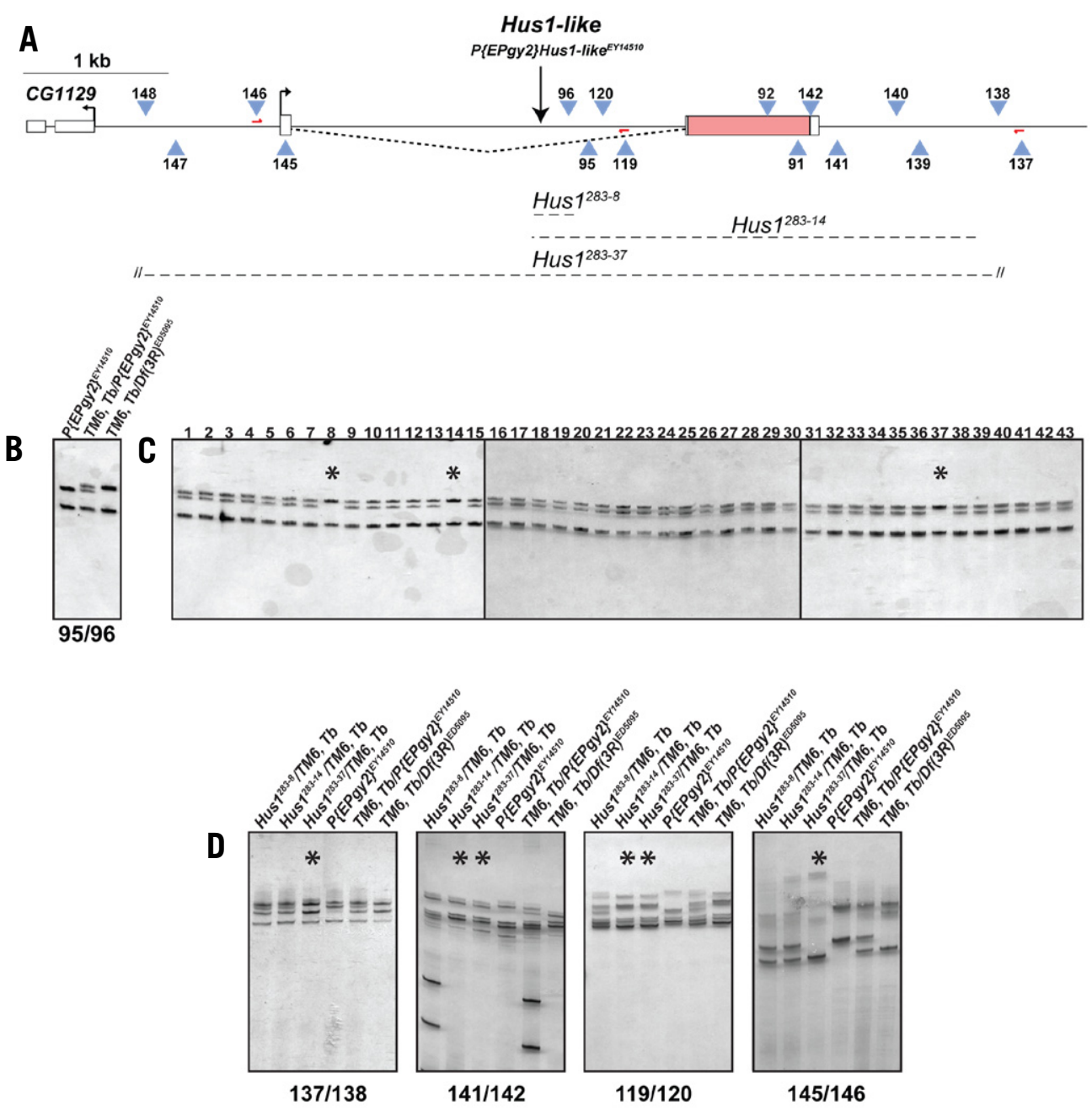

Figure 2. Characterization of deletions in the Hus1-like locus. (A) Schematic of structure of the Husl-like locus and all primer sets used in this study. Exons are boxed; non-coding regions are shown in white and coding sequences are colored. Blue triangles symbolize the primer positions for screening and mapping; red semi-arrows correspond to the primers used for sequencing the limits of deletions. The starting $P$-element is represented by a vertical arrow. Dashed lines show the deletions generated. (//) represents undetermined limit of deletion. (B) SSCP gels stained with a DNA silver staining kit showing the single-stranded secondary structure profile of the region amplified by primers 95/96 used for initial screening for imprecise excision events. Three distinct patterns are evident, corresponding to the $P$-element insertion (P\{EPgy2\}EY14510), the mapping chromosome (TM6B,Tb), and the heterozygous P\{EPgy2\}EY14510/TM6B,Tb combination. (C) SSCP gels from the screen to select imprecise excision removing the region amplified by 95/96. Precise excisions have a pattern similar to the heterozygote; imprecise excisions deleting the region of interest (indicated by an asterisk) show only the pattern of the mapping chromosome (lines \#8, \#14, and \#37). (D) SSCP gels from the mapping of the deletion carried by the lines Hus1-like283-8, Hus1-like283-14, and Hus1-like $283-37$ for the most significant regions of the Hus1-like locus. Primer sets used are indicated under each panel. Occasionally, higher mobility bands are seen in samples that are heterozygous, but not homozygous for small deletion polymorphisms (141/142), possibly due to cleavage at mismatch sites. Pertinent profiles corresponding to deletions of the region analyzed are indicated by asterisks.

DNA preparation

Individual flies were ground in $100 \mu \mathrm{L}$ solution (consisting of $10 \mathrm{mM}$ Tris- $\mathrm{Cl} \mathrm{pH}$ $8.2,1 \mathrm{mM}$ EDTA, $25 \mathrm{mM} \mathrm{NaCl}$, and 200 $\mu \mathrm{g} / \mathrm{mL}$ proteinase $\mathrm{K}$ ), incubated for $30 \mathrm{~min}$ at $37^{\circ} \mathrm{C}$, and then denatured for $2 \mathrm{~min}$ at $94^{\circ} \mathrm{C}$ to inactivate the proteinase $\mathrm{K}$. DNA preparations were stored at $-20^{\circ} \mathrm{C}$.

Polymerase chain reaction (PCR) Primers 18-22 nucleotides long with melting temperatures of $\sim 60^{\circ} \mathrm{C}$ were designed using Primer3 (v. 0.3.0) (http:// frodo.wi.mit.edu) to amplify genomic regions of $150-300 \mathrm{bp}$. Genomic sequence and primers were visualized by using GenePalette (www.genepalette.org). The sequences of all primers used in this study are available upon request.

DNA fragments from single flies were amplified as follows: $1.5 \mu \mathrm{L}$ single fly DNA, $10 \mu \mathrm{L}$ pair of primer mix $(1 \mu \mathrm{M}$ each) and $10 \mu \mathrm{L}$ Taq PCR Master Mix (Cat. no. 201445; Qiagen, Crawley, West Sussex, UK). Cycle conditions were $3 \mathrm{~min}$ at $94^{\circ} \mathrm{C}$; 34 cycles of 30 s at $94^{\circ} \mathrm{C}, 30$ s at $58^{\circ} \mathrm{C}$, and $90 \mathrm{~s}$ at $72^{\circ} \mathrm{C}$; and then a final step of $72^{\circ} \mathrm{C}$ for $10 \mathrm{~min}$. The purity of PCR products was evaluated by gel electrophoresis before analysis for polymorphisms.
Single-strand conformation polymorphism (SSCP) analysis For SSCP (Figure 1), PCR products were mixed with an equal volume of SSCP loading buffer $(0.05 \%$ bromophenol blue, $0.05 \%$ xylene cyanol, $95 \%$ formamide, $10 \mathrm{mM}$ EDTA), denatured at $95^{\circ} \mathrm{C}$ for 5 min, and then rapidly cooled on ice. Four microliters of the mix were loaded on a precast $12.5 \%$ acrylamide gel (GeneGel Excel 12.5/24 Kit, Cat. no. 17-6000-14; GE Healthcare Bio-Sciences AB; Chalfont St Giles, Buckinghamshire, UK) and run on an electrophoresis unit (Genephor, GE Healthcare) at $600 \mathrm{~V}, 25 \mathrm{~mA}, 15 \mathrm{~W}$, 
A
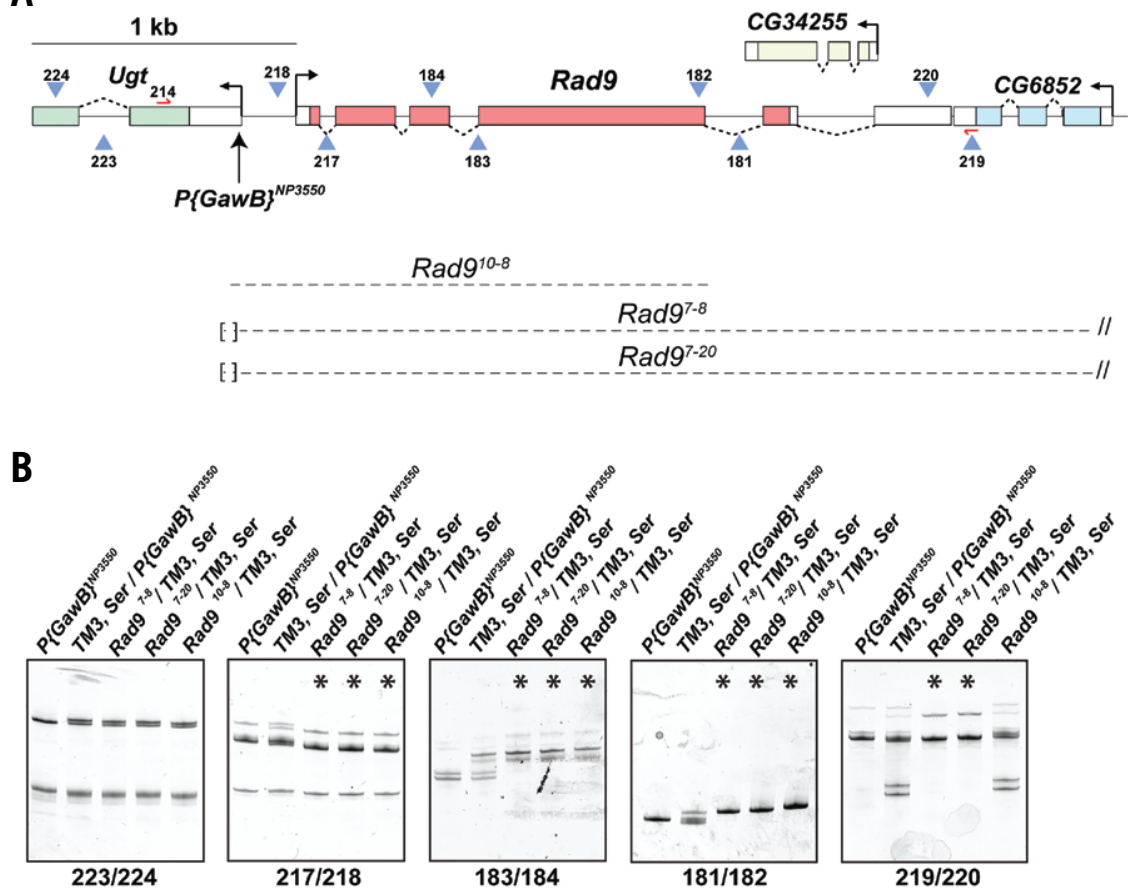

C

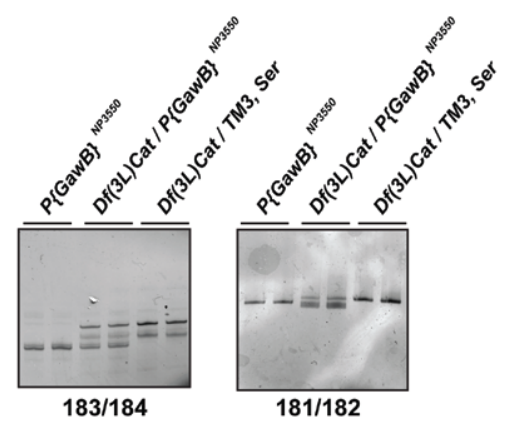

Figure 3. Structure and analysis of the Rad9 genomic locus. (A) The $P$-element insertion $P\{\text { GawB }\}^{\text {NP3550 }}$ localized in the 5'UTR of Ugt was used to generate imprecise excisions. ([.....]) represents imprecisely mapped boundaries limited by two primers sets, and (//) represents undetermined limit of deletion. Otherwise, labeling is as for Figure 2. (B) SSCP gels from the mapping of the deletion carried by the lines $\operatorname{Rad} 97-8$, $\operatorname{Rad} 97-20$ and $\operatorname{Rad} 910-8$. Non-deleted regions have a pattern similar to the heterozygote TM3,Ser /P\{GawB $\}^{N P 3550}$; excised regions exhibit only the pattern of the mapping chromosome $T M 3$, Ser (complementary to $P\{G a w B\}^{N P 3550}$ ). Primer sets tested are indicated under each panel. Profiles corresponding to deletions are indicated by an asterisk. (C) SSCP profile comparison of Rad9 amplified by $181 / 182$ and $183 / 184$. The polymorphic profile comparison of the deficiency $\mathrm{Df}(3 \mathrm{~L})$ Cat with two others polymorphic chromosomes shows that $\mathrm{Df}(3 \mathrm{~L})$ Cat does not remove the Rad9 gene. Two individual flies were tested for each genotype.

and $10^{\circ} \mathrm{C}$ for $2 \mathrm{~h}$. After electrophoresis, DNA was visualized by silver staining (Cat. no. 17-6000-30; GE Healthcare). Any cooled PAGE system may be used for SSCP analysis, and homemade silver staining solutions may be used to detect the DNA.

Polymorphism identification

To identify polymorphisms suitable for screening, we tested five different, unrelated chromosomes (including balancers) for SNP polymorphisms between the EY14510 chromosome in the Husl-like region. For homozygous-lethal balancers, we tested DNA from flies heterozygous for a deficiency covering the region of interest $\left[D f(3 R) E D_{50} 95\right]$. We examined 25 sites using pairs of PCR primers 150-300 nucleotides apart, distributed over $7.5 \mathrm{~kb}$ (Figure 2A). We focused predominantly on intronic and intergenic regions in which greater sequence variation might be expected (18), but we also occasionally found polymorphisms in exons (e.g., Figure 2A). Twelve ( 50\%) of the sites were polymorphic between EY14510 and a TM6B,Tb balancer that showed a stronger polymorphic divergence compared with the non-balancer chromosomes tested (data not shown). The use of $T M 6 B, T b$ also facilitated subsequent stock derivation.

\section{Results and discussion}

General method

Our screening and mapping process is based on using DNA polymorphisms (single nucleotide polymorphisms; SNPs) as co-dominant markers that distinguish between the chromosome with the transposable element and an unrelated chromosome. Sequencing and SNP mapping have revealed a high density $(>2$ per $\mathrm{kb}$ ) of phenotypically neutral sequence polymorphisms between different strains of $D$. melanogaster throughout the genome (18-22). The high incidence means that polymorphisms between unrelated strains are readily discovered without prior knowledge of their exact genetic backgrounds.

A large number of techniques are commonly used to detect polymorphisms including enzymatic cleavage (e.g., $\mathrm{Cel}$ I endonuclease), direct sequencing, hybridization, denaturing high-performance liquid chromatography (HPLC), ligation, and gel electrophoresis (23). In principle, any of these can be used for screening by SNP genotyping. For the purposes of this paper, we utilized SSCP because it facilitates identification of scorable SNPs (or other sequence variants) without prior knowledge of their DNA sequence, allows for rapid allelic discrimination of hundreds of samples $(24,25)$, and is an established technique for mapping point mutations in Drosophila $(23,26)$.

SSCP detects sequence variants because single-base changes cause short single-stranded DNA fragments $(\leq 300$ bp) to adopt distinct secondary structures that have different mobilities during non-denaturing gel electrophoresis (27). Under such electrophoretic conditions, DNA from heterozygous animals will generate a combination of both patterns (Figure 1A). Under optimal conditions, typically $90 \%$ of single-base polymorphisms are detected (plus essentially all small deletions and insertions) (28).

In summary (Figure 1), our screening and mapping process involved the following.

1. Identification of polymorphisms (by SSCP or other methods) between the transposon-bearing chromosome (chromosome-T) and an unrelated mapping chromosome (chromosome-M) that lie in and around the gene of interest 
(Figure 1A). If desired, this step can be carried out in parallel with step 2 to save time and avoid unnecessary fly breeding.

2. Mobilization of the transposon and selection for excision events by the loss of the transposon-associated visible marker. We used transposons carrying $w^{+}$.

3. Mating of males that have lost the transposon $\left(w^{-}\right)$to chromosome-M females for 2-5 days (Figure 1B) to allow recovery of deletion-bearing progeny in the next generation. ( $w$ - females can also be used, but are much less convenient because they have to be sacrificed for subsequent analysis.)

4. SSCP analysis of DNA from the $w^{-}$males to identify flies with imprecise excisions that extend in the desired direction (fragment B; Figure 1B). These flies lack the polymorphic allele derived from the chromosome-T (Figure 1, A and B). Crosses from precise or unwanted imprecise excisions can be discarded at this stage.

5. The remaining males are then evaluated by SSCP analysis with more distant probes to identify individuals in which the deletion extends into the ORF of interest but not into either flanking gene (fragments A, C, and D; Figure 1B).

6 . The remaining flies reflect single gene deletions, whose exact extents can be determined by PCR amplification and DNA sequencing of the junction fragment, usually using primers made previously for polymorphism analysis.

\section{Generating a single-gene}

deletion of Hus1-like

We first used this approach to screen for small deletions affecting the Husl-like locus (82D6 on chromosome 3R), which encodes the conserved Husl cell cycle checkpoint protein. Hus1 is a component of the 9-1-1 complex that mediates cellular responses to DNA damage (29). Husl-like mutants were not available when our experiments were started, but a $P\left(w^{+}\right)$ transposon (P\{EPgy2\}HUS1-like EY14510) had been mapped to the first intron of Husl-like, $1 \mathrm{~kb}$ upstream of the start of the second exon that contains the entire coding sequence of Husl-like (Figure 2A).

Husl is a non-essential gene in yeast (30), but essential during development in mouse (31). Flies homozygous for $P\{E P g y 2\}$ HUS1-likeEY14510 (and other insertions tested near the gene) are viable and fertile, and so it was unclear what phenotype to expect for Husl-like mutations, although subsequent work has shown that Drosophila Husl-like null mutant flies are viable but poorly female-fertile (32).
To screen and map the deletion of Husl-like, we used eight polymorphisms between the P\{EPgy2\}HUS1-like EY14510 chromosome and a $T M 6 B, T b$ balancer (see "Materials and methods" section; Figure 2A). Imprecise excisions were generated by crossing $w$; Husl-like EY14510 flies to $w^{-} ; M K R S, P\{\Delta 2-3\} / T M 6 B, T b$ flies that express high levels of P-transposase (33). Male progeny exhibiting mosaic eyes were crossed to $T M 6 B, T b$ females and heterozygous TM $6 B$ offspring were screened for the loss of the $w^{+}$marker. Individual $w^{-}$males were mated for propagation, then removed after $2-5$ days (after larval activity in the food was evident) and their DNA prepared for SNP analysis (see "Materials and methods" section).

To screen for imprecise excision events that remove the open reading frame (ORF) of Husl-like, we amplified a polymorphic fragment situated 130 bp downstream of the $P$-element insertion site for SSCP analysis (primers 95/96; Figure 2A). Flies that display both parental electrophoretic variants retain this region (and, by inference, the complete Husl-like ORF), whereas imprecise excision events extending toward the Husl-like ORF show only the balancer variant (Figure 2B). From the $82 w^{-}$flies screened, three lacked the variant corresponding to the transposonbearing chromosome [flies Husl-like ${ }^{283-8}$, Husl-like 283-14, and Husl-like 283-37; Figure 2C (lanes \#8, \#14, and \#37)], indicating that these flies bear deletions that extend in the appropriate direction (Figure 2, B and $\mathrm{C}$ ).

The deletions carried by these three lines were mapped further using other primer pairs to refine the boundary limits (Figure 2D and data not shown).

Husl-like $e^{283-8}$ is deleted at the 95/96 site, but retains sites $119 / 120$ and 145/146. This deletion lacks some intronic sequence and possibly the first exon of Husl-like but does not extend into the coding sequence (Figure 2A and D). Husl-like 283-14 lacks all sites including and between 95/96 and $139 / 140$, but retains $137 / 138$ and $145 / 146$ indicating that the entire Husl-like coding sequence is removed, and that the deletion does not reach the adjacent genes (Figure 2, A and D). Husl-like 283-8 and Husl-like 283-14 are both homozygous viable and show no obvious phenotypes. However, the fertility of female homozygotes for the null alleles Husl-like $283-14$ is affected, consistent with the recently described Husl-like null phenotype (32). Husl-like $e^{283-37}$ is a large deletion $(>7.5 \mathrm{~kb})$, that extends on both sides of the insertion, removing the entirety of the Husl-like locus and several adjacent genes lying 5'
WEW BRANDplates Mieroplates trim

Germany's precision lab plastics manufacturer announces a comprehensive line of microplates for general, cell culture and immunology applications. The line includes plates with distinctive physical treatments that eliminate refrigeration and shelf-life concerns. All are SBS-formatted.
- 8 different surface treatments
- 96-, 384- and 1536-well

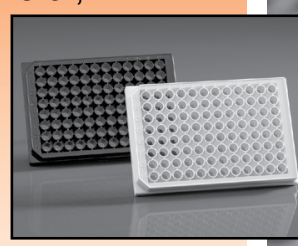

- Transparent, white, black, clear bottom and UV-transparent

- C, F, V and U-shaped wells

- Standard- and low-volume

- Bar-coding on request

- Samples available

Visit our booth \#265 at Lab Automation!

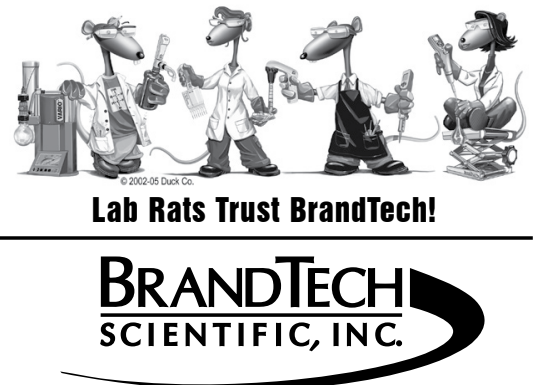

Toll Free (888) 522-2726 www.brandtech.com 
and 3 ' (data of extended SSCP analysis not shown; Figure 2, A and D). Unlike the former two lines, Husl-like $283-37$ is homozygous lethal.

We defined the exact breakpoints of the two deletions that potentially affect Husl-like (Husl-like $e^{283-8}$ and Husl-like $283-14$ ) by sequencing the products of PCR amplification using flanking primers (119/146 and 137/146, respectively) (Figure 2A). This showed that Husl-like $e^{283-14}$ is a deletion of $3.15 \mathrm{~kb}$, which removes the second exon of Husl-like (containing the entire coding sequence of the gene) without affecting the neighboring genes (Figure 2A). By contrast, Husl-like $e^{283-8}$ is a very small deletion that removes $0.3 \mathrm{~kb}$ of the first intron (Figure $2 \mathrm{~A}$ ).

Altogether, mating and screening the $w^{-}$flies took $<8$ days (i.e., less than a single generation and before offspring of the excisionbearing males had emerged). Thus, undesired excision events could be discarded without having to be established and maintained as stocks. The whole process, from establishing the excision cross and polymorphism discovery to identification of the desired deletion mutant, took less than a month.

Generating a single-gene deletion of the Rad9 locus We tested the robustness of our SNP/SSCP method by generating deletions for a second gene, $\operatorname{Rad} 9$, that encodes another component of the $9-1-1$ complex involved in the DNA damage signaling pathway (29).

We started from a $P$-element insertion $(P\{G a w B\} N P 3550)$ that lies 240 bp upstream of the Rad9 promoter (75E2-75E3 on

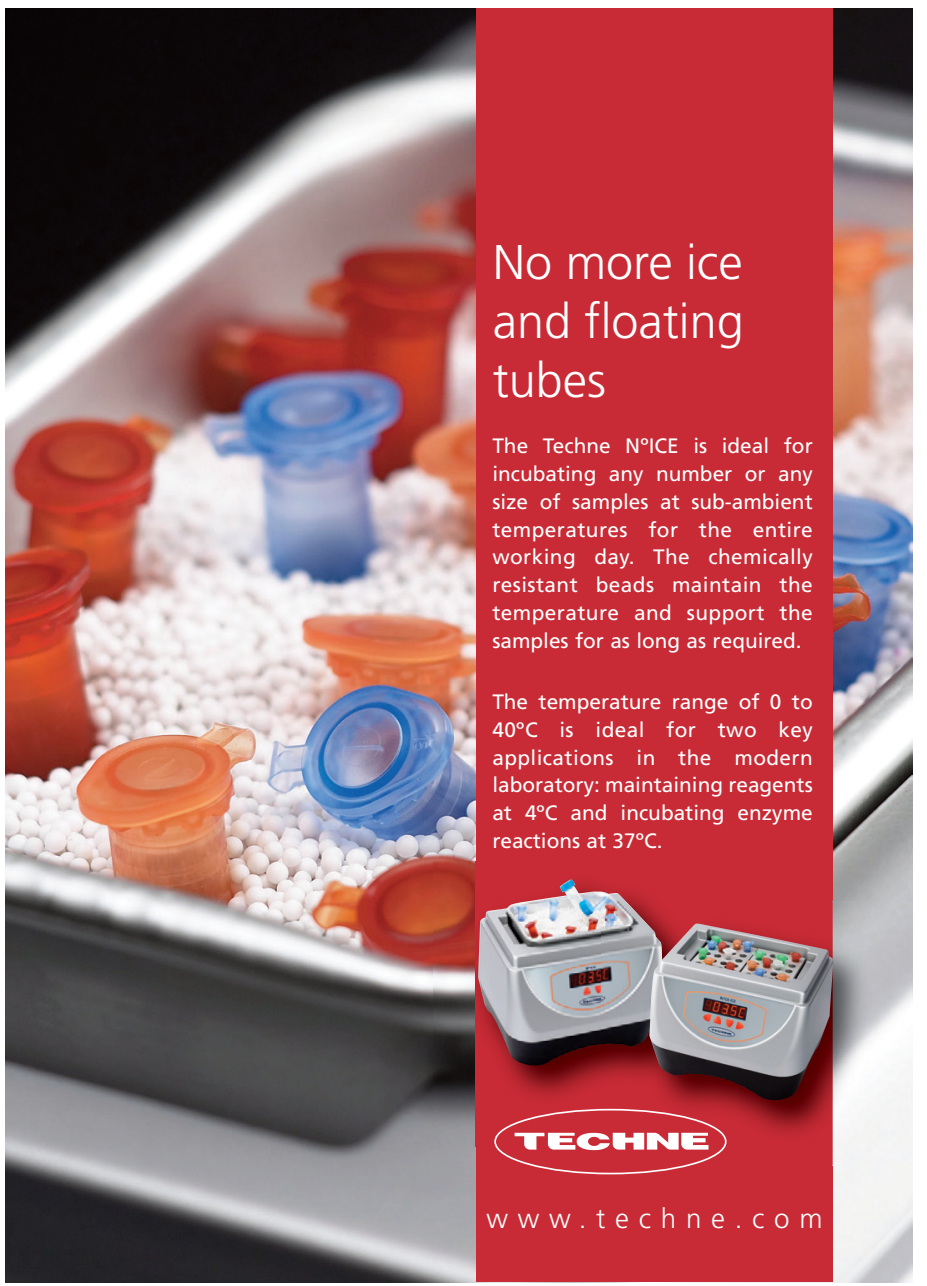

chromosome 3L), and 30 bp into the first exon of the adjacent gene (UDP glucose-glycoprotein glucosyltransferase, or $U g t$ ) (Figure 3A). From twelve sites in and around the Rad9 locus tested by SSCP, we used five polymorphisms between the $P\{G a w B\} N P 3550$ chromosome and a TM3, Ser balancer. We screened $200 w^{-}$males using a polymorphic fragment amplified by primers $217 / 218$, and recovered three imprecise excision events extending toward the Rad9 ORF (flies $\operatorname{Rad}^{7-8}, \operatorname{Rad}^{7-20}$, and $\operatorname{Rad} 9^{10-8}$; Figure 3A).

We refined our mapping of the limits of these three deletions using four other primer sets (Figure 3B), then DNA sequencing, as for Husl-like above. Two of the deletions ( $R$ ad97-8 and Rad97-20) retain the 223/224 sites within Ugt, but their $3^{\prime}$ ends extend beyond the most distant polymorphism characterized (lying more than $30 \mathrm{~kb}$ downstream of Rad9; data not shown), indicating that these deletions remove several additional, distal genes (Figure $3, \mathrm{~A}$ and $\mathrm{B})$. The third deletion ( $\left.\operatorname{Rad} 9^{10-8}\right)$ is homozygous viable and lacks the $217 / 218,183 / 184$, and $181 / 182$ sites but retains the 223/224 and 219/220 sites (Figure 3, A and B). After PCR and sequencing, we determined that $\operatorname{Rad} 910-8$ is deleted for most of the coding sequence without affecting the coding sequence of the overlapping $3^{\prime}$ gene $C G 34255$ and the coding region of the neighboring $5^{\prime}$ gene $U g t$ (although, like the parental chromosome, expression of the latter is reduced; not shown). Again, mating and screening the $w^{-}$flies to recover this deletion took less than 10 days.

We also used SSCP profiling to establish if $D f(3 L) C a t$, a chromosomal deletion generated by $\gamma$-ray that has been genetically mapped to roughly cytological position $75 \mathrm{C} 1-75 \mathrm{C} 2$ to 75F1 (http://flybase.org), overlaps the Rad9 locus. We compared SNPs between $P\{G a w B\}^{N P 3550}, D f(3 L) C a t / P\{G a w B\}^{N P 3550}$, and Df(3L)Cat/TM3, Ser (Figure 3C). Heterozygosity for 183/184 and $181 / 182$ shows that this deficiency does not remove the $R$ adg gene (Figure 3B). Hence, this method can also be used to characterize the extent of independently generated deficiencies.

We have demonstrated the efficiency and convenience of sequence polymorphisms used as co-dominant markers to screen for null alleles of previously uncharacterized genes, independently of the mutant phenotype. We were able to rapidly recover singlegene deletions in two independent loci in the Drosophila genome, and also identified several other larger deletions that would have been difficult or impossible to detect using the traditional PCR method, and which might prove useful in other contexts (e.g., for generating deletions of small overlapping regions).

Our method uses DNA polymorphisms as co-dominant markers to distinguish between the chromosome carrying the transposable element and its unrelated homolog to identify all deletions in the polymorphic region. Imprecise excision events are identified by selective loss of polymorphic alleles associated with the strain bearing the transposable element. Although polymorphisms arise more frequently in intronic and intergenic regions (19), we also found polymorphisms in exons. The high density of phenotypically neutral sequence polymorphisms in Drosophila $(>2 / \mathrm{kb})(18-22)$, allows us to delimit both breakpoints of each deletion to within $0.5-1 \mathrm{~kb}$, thereby rapidly identifying the events that affect only the gene of interest. Although we compared polymorphisms using SSCP in this study, similar results would have been obtained using alternative methods for SNP detection (e.g., sequencing and denaturing HPLC).

Polymorphism screening has several major advantages over other strategies for mapping mutations derived from imprecise excision, including its ability to detect all relevant events without phenotypic bias. Also, it uses pretested primers and reaction conditions for reliable amplification of crude genomic DNA extracted directly from single flies. By contrast, classical PCR screens 
depend on untested primer pairs (since they are designed not to amplify across the transposon) and generate fragments of unpredictable sizes that often necessitate multiple screens and gel runs per excision event. Thus, the classical PCR method usually requires better-quality DNA extracted from multiple flies, resulting in additional fly husbandry costs and effort compared with our strategy.

The ratio of imprecise to precise excision events of transposable elements is variable (usually between 1:5 and 1:20) (11,34). Of these, only a proportion will extend toward and into the gene of interest. In our experiments, we found that $1.5-3.6 \%$ of excision events extended to a nearby polymorphic site. The frequency of such excisions can be increased by inhibiting the pairing between the transposable element chromosome and its homolog via a chromosome deficiency (14), or by screening in a mus309 mutant background that lacks the Bloom syndrome helicase, thus increasing the frequency and size of the deletions generated from $P$-element and minos excisions (15). However, both these schemes involve considerable extra breeding. We screened up to 100 flies with little effort, and could have readily scaled up the experiments in order to identify rarer events.

We have shown that codominant polymorphism screening also enables mapping of pre-existing deletions $[D f(3 L)$ Cat; Figure 3C]. Indeed, it can easily be adapted for use in other genetic model organisms for which it is possible to generate imprecise excisions by mobilizing transposable elements $(35,36)$ or by $\mathrm{x}$-rays. SNPs are frequent in both the mouse [1 per $\sim 300$ nucleotides (37)] and zebrafish [>1 per kb (38)]. Furthermore, screening for sequence polymorphisms facilitates the identification of targeted mutations generated by engineered zincfinger nucleases $(39,40)$ (E.V. and D.I.-H., unpublished observations).

\section{Acknowledgments}

We thank Rippei Hayashi, Ned Hoyle, Michael Stauber, Mark Wainwright, and Krzysztof Wicher for comments on the manuscript and helpful discussions. This work was supported by Cancer Research UK. E.V. was the recipient of a Federation of European Biological Sciences long-term fellowship.

\section{Competing interests}

The authors declare no competing interests.

\section{References}

1. Adams, M.D. and J.J. Sekelsky. 2002. From sequence to phenotype: reverse genetics in Drosophila melanogaster. Nat. Rev. Genet. 3:189-198.

2.Venken, K.J. and H.J. Bellen. 2005. Emerging technologies for gene manipulation in Drosophila melanogaster. Nat. Rev. Genet. 6:167-178.

3. Huang, J., W. Zhou, A.M. Watson, Y.N. Jan, and Y. Hong. 2008. Efficient ends-out gene targeting in Drosophila. Genetics 180:703707.

4.Dietzl, G., D. Chen, F. Schnorrer, K.C. Su, Y. Barinova, M. Fellner, B. Gasser, K. Kinsey, et al. 2007. A genome-wide transgenic RNAi library for conditional gene inactivation in Drosophila. Nature 448:151-156.

5. Carroll, D., K.J. Beumer, J.J. Morton, A. Bozas, and J.K. Trautman. 2008. Gene targeting in Drosophila and Caenorhabditis elegans with zinc-finger nucleases. Methods Mol. Biol. 435:63-77.

6. Beumer, K., G. Bhattacharyya, M. Bibikova, J.K. Trautman, and D. Carroll. 2006. Efficient gene targeting in Drosophila with zinc-finger nucleases. Genetics 172:2391-2403.

7. Thibault, S.T., M.A. Singer, W.Y. Miyazaki, B. Milash, N.A. Dompe, C.M. Singh, R. Buchholz, M. Demsky, et al. 2004. A complementary transposon tool kit for Drosophila melanogaster using $P$ and piggyBac. Nat. Genet. 36:283-287.

8. Parks, A.L., K.R. Cook, M. Belvin, N.A. Dompe, R. Fawcett, K. Huppert, L.R. Tan, C.G. Winter, et al. 2004. Systematic generation of high-resolution deletion coverage of the Drosophila melanogaster genome. Nat. Genet. 36:288-292.

9. Voelker, R.A., A.L. Greenleaf, H. Gyurkovics, G.B. Wisely, S.M. Huang, and L.L. Searles. 1984. Frequent imprecise excision among reversions of a P element-caused lethal mutation in Drosophila. Genetics 107:279294.

10. Bellen, H.J., R.W. Levis, G. Liao, Y. He, J.W. Carlson, G. Tsang, M. Evans-Holm, P.R. Hiesinger, et al. 2004. The BDGP gene disruption project: single transposon insertions associated with $40 \%$ of Drosophila genes. Genetics 167:761-781.

11. Ryder, E. and S. Russell. 2003. Transposable elements as tools for genomics and genetics in Drosophila. Brief. Funct. Genomics Proteomics 2:57-71.

12.Metaxakis, A., S. Oehler, A. Klinakis, and C. Savakis. 2005. Minos as a genetic and genomic tool in Drosophila melanogaster. Genetics 171:571-581.

13.Johnson-Schlitz, D.M. and W.R. Engels. 1993. P-element-induced interallelic gene conversion of insertions and deletions in Drosophila melanogaster. Mol. Cell. Biol. 13:7006-7018.

14. Engels, W.R., D.M. Johnson-Schlitz, W.B. Eggleston, and J. Sved. 1990. Highfrequency $\mathrm{P}$ element loss in Drosophila is homolog dependent. Cell 62:515-525.

15. Witsell, A., D.P. Kane, S. Rubin, and M. McVey. 2009. Removal of the bloom syndrome DNA helicase extends the utility of imprecise transposon excision for making null mutations in Drosophila. Genetics 183:11871193.

\section{Why do we invest \\ somuch time in}

development and QC of

our ELISAs?

Because

quality, reproducible

and accurate

results

matter.

Take the ELISA challenge.

mdbioproducts.com/ELISAchallenge 
